\title{
Vacinação em Pacientes Imunossuprimidos e com Doenças Reumatológicas Auto-Imunes
}

\section{Vaccination for Immunocompromised Patients and Patients with Autoimmune Rheumatic Diseases}

\author{
Karine Rodrigues da Luz(1), Deborah Colucci Cavalcante de Souza ${ }^{(2)}$, Rozana Mesquita Ciconelli( ${ }^{(3)}$
}

\begin{abstract}
RESUMO
A vacinação é uma das formas de prevenção para doenças infecciosas. Pacientes com doenças reumatológicas apresentam uma maior prevalência de doenças infecciosas quando comparados com a população em geral, seja devido à deficiência imune da doença de base ou pelo uso de terapia imunossupressora. Portanto, a vacinação é uma medida eficaz para a redução da morbidade e mortalidade nesses pacientes. O objetivo deste artigo é mostrar a segurança e eficácia das vacinas em pacientes imunossuprimidos e com doenças reumatológicas auto-imunes. De um modo geral, as vacinas de bactérias e vírus mortos são seguras em pacientes com doenças reumatológicas, mesmo em uso de terapias imunossupressoras. A vacinação é eficaz para a grande maioria dos pacientes, sendo que uma pequena parcela destes não apresentam resposta imunológica satisfatória pós-imunização. Vacinas com bactérias ou vírus vivos atenuados são, em geral, contra-indicadas em pacientes imunossuprimidos.
\end{abstract}

Palavras-chave: vacinação, doenças reumatológicas, imunossuprimidos.

\section{INTRODUÇÃO}

Vacinação é a exposição deliberada, por injeção, ingestão ou inalação de um produto não-tóxico que estimula o indivíduo a produzir anticorpos ${ }^{(1)}$. Se o indivíduo é subseqüentemente exposto ao patógeno contra o qual foi vacinado, a exposição resulta em uma resposta secundária que inclui a proliferação de células B e formação de anticorpos, que protege o indivíduo de desenvolver a doença.

A prevenção de doenças infecciosas é de extrema importância em pacientes com imunidade prejudicada, pois a infecção causa grande morbidade e mortalidade nesses

\begin{abstract}
Vaccination is a way to prevent infectious diseases. Infections are more prevalent among patients with rheumatic diseases when compared to the general population. This higher prevalence may be due to an immune system deficiency associated with the underlying condition or to the use of immunessupressive therapy. Vaccination is an effective measure to reduce morbidy and mortality in these patients. The aim of this article is to review safety and efficacy issues concerning different vaccines for immunocompromised patients and patients with autoimmune rheumatic diseases. In general, vaccines with killed bacteria or virus are safe for patients with autoimmune rheumatic diseases, even when they are taking immunessupressive therapy. The vaccination is effective to most patients because they usually accomplish an effective immune response. Administration of attenuated vaccines with live bacteria or virus to immunocompromised patients is contraindicated.
\end{abstract}

Keywords: vaccination, rheumatic disesases, immunosupressive therapy.

pacientes. Os pacientes com doenças reumatológicas, principalmente de natureza auto-imune, apresentam níveis variados de imunossupressão com alterações distintas no sistema imune, dependendo da condição de base e do uso de determinada terapia ${ }^{(2)}$.

Em uma coorte de 266 pacientes com lúpus eritematoso sistêmico (LES) seguidos por 20 anos, 23,3\% destes apresentaram infecção e 30 mortes tiveram como principal causa a infecção ${ }^{(3)}$. Portanto, a vacinação de pacientes adultos com doenças auto-imunes representa uma estratégia para diminuir o risco de infecções. No entanto, algumas vacinas oferecem risco de exacerbação da doença auto-imune.

Recebido em 22/02/06. Aprovado, após revisão em 16/08/06.

Universidade Federal de São Paulo (Unifesp), Escola Paulista de Medicina, Departamento de Medicina Interna, Disciplina de Reumatologia.

1. Médica reumatologista pós-graduanda (Mestrado) da Disciplina de Reumatologia da Escola Paulista de Medicina - Unifesp

2. Médica reumatologista pós-graduanda (Mestrado) da Disciplina de Reumatologia da Escola Paulista de Medicina - Unifesp.

3. Professora afiliada da Disciplina de Reumatologia da Escola Paulista de Medicina - Unifesp.

Endereço para correspondência: Rozana Mesquita Ciconelli, Rua Botucatu, 740, $3^{\circ}$ andar, V. Clementino, CEP 04023-062, telefone (11) 5579-6665, fax: (11) 5576-4239, e-mail: rozana@reumato.epm.br 
O objetivo deste artigo é fazer uma revisão sobre a segurança e eficácia das vacinas em pacientes com doenças reumatológicas e também seus efeitos adversos, incluindo a possibilidade de exacerbação da doença reumatológica.

\section{TIPOS DE VACINAS}

As vacinas contêm diferentes componentes que as tornam mais ou menos seguras para uso nesses pacientes, sendo classificadas, segundo esses componentes, em três tipos: a) bactérias ou vírus mortos; b) bactérias ou vírus vivos atenuados; e c) proteínas ou açúcares extraídos de bactérias ou vírus ou sintetizados em laboratório. Toxóides usados para imunização ativa são toxinas bacterianas modificadas de modo a se tornarem não-tóxicas.

As vacinas de bactérias ou vírus mortos são muito seguras, por exemplo a vacina contra Haemophilus influenzae. Também as vacinas compostas por proteínas ou açúcares são, em geral, seguras.

Quando administradas, as vacinas de bactérias ou vírus atenuados, por apresentarem vírus ou bactéria fracamente vivos, infectam os pacientes e os induzem a formar anticorpos (Ac), os quais os protegerão de organismos mais virulentos. Esse tipo de vacina, em geral, é segura para indivíduos saudáveis. No entanto, por produzirem uma infecção mínima, elas podem, em pessoas nas quais o sistema imune esteja prejudicado, por exemplo em pacientes recebendo corticosteróides (CE) ou outras drogas imunossupressoras, provocar infecção incontrolável.

No Brasil, o Ministério da Saúde, a partir de 2004, passou a adotar três calendários obrigatórios de vacinação no território nacional: o Calendário Básico da Vacinação da Criança, o Calendário da Vacinação do Adolescente e o Calendário de Vacinação do Adulto e do Idoso $^{(4)}$.

\section{VACINAÇÃO EM PACIENTES COM DOENÇAS AUTO-IMUNES}

O British Society of Rheumatology (BSR) Clinical Affairs Comitee propôs um guideline para vacinação em pacientes imunossuprimidos, com doenças reumatológicas, tratados com drogas citotóxicas ou esteróides, em que definiu como dose baixa de corticosteróide 10 $\mathrm{mg} / \mathrm{d}$ e dose alta como $\geq 20 \mathrm{mg} / \mathrm{d}$. O guideline do Departamento de Saúde do Reino Unido recomenda que pacientes recebendo altas doses de CE não devem receber vacinas de vírus vivo atenuado, necessitando aguardar três meses após a suspensão do tratamento para, então, receber essas vacinas ${ }^{(5)}$.
A imunização em pacientes com doença reumatológica suscita questões quanto à possibilidade de a vacinação desencadear ou piorar uma doença reumatológica, à sua eficácia e aos possíveis riscos da vacinação nesses pacientes.

O mecanismo hipotético explicativo mais atraente pelo qual um agente infeccioso pode deflagrar uma resposta auto-imune é a mímica molecular. De acordo com essa hipótese, determinantes antigênicos dos microorganismos podem ser reconhecidos pelo sistema imune do hospedeiro como sendo semelhantes aos seus próprios determinantes antigênicos. É possível que qualquer microorganismo que expresse um epítopo que possa servir como mímica molecular para um auto-antígeno seja capaz de induzir resposta auto-imune. Portanto, teoricamente, a vacinação poderia induzir reações auto-imunes e mesmo doenças auto-imunes. Existem relatos de várias dessas doenças que se iniciaram após o uso de vacinas. A vacinação pode, portanto, ter efeitos auto-imunes e pode mesmo deflagrar uma doença auto-imune, embora isso seja raro $^{(6)}$.

Algumas vacinas, especialmente a vacina contra rubéola, podem causar uma artrite que pode durar várias semanas e desaparecer espontaneamente. Vacinação contra o vírus da influenza pode raramente induzir à doença neurológica, como a síndrome de Guillain-Barré. A Tabela 1 mostra as doenças auto-imunes relatadas após vacinação.

TABela 1

DOENÇAS AUTO-IMUNES RELATADAS APÓS VACINAÇÃO

\begin{tabular}{lc}
\hline Doença & Tipo de vacina \\
\hline Lúpus eritematoso sistêmico & Hepatite B, tétano e antraz \\
Artrite reumatóide & Hepatite B, tétano, tifóide/paratifóide, SCR \\
Síndrome de Sjögren & Hepatite B \\
Esclerose múltipla & Hepatite B \\
Artrite reativa & BCG, tifóide, DPT, SCR, hepatite B, influenza \\
Poli/dermatomiosite & BCG, difteria, DPT \\
Poliarterite nodosa & Influenza, pertussis, hepatite B \\
Arterite de Takayasu & Hepatite B \\
Síndrome de Guillain-Barré & Influenza, pólio, tétano \\
Diabetes melito tipo I & Hepatite B \\
Trombocitopenia idiopática & SCR, hepatite B \\
\hline
\end{tabular}

Estudos sobre imunização em pacientes com LES mostram diferentes resultados quanto ao surgimento de flares pós-vacinação.

Battafarano $e t a l .{ }^{(7)}$ não observaram alteração significante na atividade da doença após vacinação de 73 pacientes contra pneumococos, Haemophilus influenzae tipo B e toxóide tetânico. Já Brow et al ${ }^{(8)}$, na imunização contra poliomielite de 73 pacientes com LES, em uma campanha em Israel, relatam flares em $5 \%$ deles. Outras vacinas que também podem induzir ou reativar o LES incluem as vacinas contra influenza, hepatite $\mathrm{B}$ e tétano ${ }^{(9,10)}$. 
A imunização contra a hepatite $\mathrm{B}$ em pacientes com LES deve ser feita apenas nos indivíduos expostos ao risco de infecção. Em geral, as vacinas se mostram eficazes, embora esses pacientes apresentem uma resposta imune à vacinação menor do que em controles saudáveis ${ }^{(11)}$.

A artrite reumatóide e outras doenças reumatológicas também podem ter seu início ou reativação após vacinação, especialmente após o uso das vacinas contra a hepatite $\mathrm{B}^{(12,13)}$.

Vacinas com bactérias ou vírus mortos e vacinas contendo componentes imunizantes ou toxóides são seguras em indivíduos imunossuprimidos ${ }^{(5)}$.

\section{VACINAÇÃO EM PACIENTES IMUNOSSUPRIMIDOS}

\section{VACINAS CONTRA DOENÇAS BACTERIANAS}

BCG - A vacina BCG (bacilo de Calmette e Guerin) é administrada com a finalidade de prevenir as formas graves da tuberculose (miliar e meníngea). A vacina BCG é preparada com bacilos vivos, a partir de cepas atenuadas do Mycobacterium bovis.

Constituem-se contra-indicação para a vacinação com BCG os portadores de HIV, positivos sintomáticos, bem como outras situações gerais de contra-indicação para todo imunobiológico, quais sejam: presença de imunodeficiência congênita ou adquirida, presença de neoplasia maligna, vigência de tratamento com corticóides em dose imunossupressora (equivalente a $2 \mathrm{mg} / \mathrm{kg} /$ dia para criança ou $\geq 20 \mathrm{mg}$ /dia para adulto, por mais de uma semana), vigência de outras terapêuticas imunodepressoras como quimioterapia antineoplásica, radioterapia ou outras e hipersensibilidade aos componentes da fórmula.

Toxóide tétano/difteria (dT) - O tétano e a difteria são considerados doenças raras, porém ainda ocorrem em populações que não completaram a imunização primária.

A vacina dT contém o toxóide do tétano e diftérico. É composta por toxinas bacterianas inativadas imunogênicas e não causam doença ativa. Está indicada para indivíduos com mais de 7 anos que não tiveram imunização prévia adequada com a DTP (difteria, tétano, pertussis) ou com a tetra (DTP $+\mathrm{Hib}=\mathrm{DTP}+$ Haemophilus influenzae tipo B). Devem ser administradas três doses seriadas (0,2 e 8 meses). Na idade adulta, a recomendação é administrar o toxóide a cada dez anos em adultos que receberam a imunização primária. Se há risco infeccioso para o tétano, deve-se administrar o toxóide tetânico, caso este não tenha sido administrado nos últimos cinco anos. Deve ser aplicada a gamaglobulina específica simultaneamente com a vacina, caso o paciente não saiba quando ocorreu a imunização primária ${ }^{(14)}$.

Os efeitos adversos do toxóide são os sintomas nos locais de aplicação (dor e inflamação) e febre. As contra-indicações são hipersensibilidade grave ou reações neurológicas a doses prévias.

Symmons et al..$^{(15)}$ constataram que, dentre 588 pacientes notificados no Norfolk Arthritis Register, 19 (3\%) tinham apresentado artrite após seis semanas da imunização com toxóide tetânico. Destes, 12 preencheram os critérios de 1987 do American College of Rheumatology para o diagnóstico de artrite reumatóide, quando vistos pela primeira vez. Oito apresentaram fator reumatóide positivo e cinco desenvolveram erosões radiográficas no primeiro ano. Dois outros pacientes haviam sido imunizados, um contra influenza e outro contra hepatite B, porém estes não satisfizeram os critérios da ACR para artrite reumatóide; a apresentação desses sintomas pós-vacinação mostra haver uma aparente associação entre imunização e desenvolvimento de $\operatorname{artrite}^{(15)}$. Outro estudo sobre possível associação entre vacinação e desenvolvimento de artrite não identificou diferenças clínicas entre pacientes com poliartropatia inflamatória com e sem vacinação prévia ao início da atividade inflamatória ${ }^{(16)}$.

A imunização com toxóide tetânico foi segura em pacientes com LES, conforme estudo de Battafarano et al.(7), e $90 \%$ dos pacientes vacinados apresentaram títulos de anticorpos protetores.

Adultos com doenças reumatológicas devem receber vacinação contra tétano e difteria de acordo com a recomendação para adultos saudáveis, pois esta é considerada segura e eficaz ${ }^{(2)}$.

Vacina polissacarídeo pneumocócica $(23-\mathrm{V})$ - A cada ano, a doença pneumocócica é responsável por 3 mil casos de meningite, 50 mil casos de bacteremia, 500 mil casos de pneumonia e 7 milhões de casos de otite média ${ }^{(17)}$.

Pessoas morrem mais de infecções pneumocócicas do que de outras doenças preveníveis por vacinas. A septicemia, a meningite e outras complicações da infecção pneumocócica são causas significativas de doença grave e morte em pacientes esplenectomizados, pacientes com lúpus eritematoso sistêmico, pacientes em uso de corticosteróides a longo prazo e outros indivíduos imunocomprometidos ${ }^{(2)}$.

Existem, atualmente, duas vacinas pneumocócicas polissacarídeas viáveis. Ambas contêm antígenos polissacarídeos purificados de 23 sorotipos pneumocócicos que são responsáveis por $85 \%$ a $90 \%$ das infecções invasivas ${ }^{(17)}$. 
A dose é única, intramuscular ou subcutânea com um reforço após cinco anos. Está indicada, entre outras situações, para crianças a partir dos 2 anos e adultos com imunodeficiência congênita ou adquirida e em pacientes recebendo corticosteróides a longo prazo.

Os efeitos adversos mais comuns são sintomas no local da aplicação, febre, cansaço e mialgia. Em pacientes adultos com doenças auto-imunes, a vacina pneumocócica pode produzir imunogenicidade e duração de imunidade reduzida. Estudo com 73 pacientes com LES submetidos à vacina pneumocócica mostrou resposta ineficiente em $47 \%$ dos pacientes, avaliada pela dosagem de anticorpos protetores 12 semanas após o procedimento. Além do mais, houve uma tendência à diminuição de resposta em pacientes com doença ativa, tratados com drogas imunossupressoras, porém a atividade da doença não foi afetada pela vacinação ${ }^{(7)}$.

O estudo de Elkayam et al. ${ }^{(18)}$ avaliou 42 pacientes com AR, 24 pacientes com LES e 20 controles vacinados com a vacina pneumocócica 23 -valente. Os pacientes com LES e $\mathrm{AR}$, enquanto grupos, apresentaram boa resposta à vacina pneumocócica após um mês, semelhantemente ao grupocontrole; porém, 20,8\% e 33,3\% dos pacientes com LES e $\mathrm{AR}$, respectivamente, apresentaram individualmente uma resposta deficiente de anticorpos, respondendo a nenhum ou apenas a um dos sete polissacarídeos pneumocócicos testados. Nenhuma medida clínica ou laboratorial se correlacionou com a resposta diminuída de anticorpos nas duas doenças.

Tarjan et al. ${ }^{(19)}$ acompanharam 18 pacientes com LES e nove controles que receberam a vacina pneumocócica. Entre os pacientes com LES, a dose média de metilprednisolona era de $5,9 \mathrm{mg} / \mathrm{d}$, sendo que dois pacientes faziam uso de droga imunossupressora (azatioprina 100 $\mathrm{mg} / \mathrm{d}$ ). Vinte e oito dias após a vacinação, não se observou reativação da doença. Observou-se menores níveis de Ac nos pacientes com LES do que nos pacientes-controles. Sugere-se, então, que, se a resposta a Ac for ineficiente e a doença se mantiver inativa com a vacinação, os pacientes portadores de LES poderão receber as vacinas desenvolvidas mais recentemente, as quais são mais imunogênicas. O estudo de O'Dell et al. ${ }^{(20)}$ mostrou uma resposta diminuída à vacina pneumocócica em pacientes que estavam sendo tratados com metotrexato (MTX). Nesse estudo, idade, sexo, duração da doença, medidas de atividade da doença e o uso de drogas imunossupressoras (corticosteróides e azatioprina), sulfassalazina e agentes antimaláricos não se associaram de forma significativa com a resposta de anticorpos. É importante ressaltar que a dose média de CE entre os pacientes com AR era menor do que $10 \mathrm{mg} / \mathrm{dia}$ de prednisona (apenas $11 \%$ em uso $>10 \mathrm{mg} /$ dia) e apenas um paciente fora tratado com ciclofosfamida $(61 \%$ estavam em uso de MTX e 16\%, em uso de azatioprina).

Embora haja relatos de que a vacinação contra doença pneumocócica seja segura e eficaz em pacientes em uso de agentes biológicos (drogas anti-TNF), Elkayam et al..$^{(21)} \mathrm{em}$ um estudo no qual foram dosados os níveis de IgG antipneumocócica, um mês após a vacinação, em 16 pacientes tratados com anti-TNF (11 com artrite reumatóide e 5 com espondilite anquilosante) e, em 17 pacientes controle pareados para a idade tratados como outros agentes imunossupressores, encontraram uma tendência de redução nos níveis de Ac, para todos os sorotipos, exceto para o sorotipo 14, nos pacientes em uso do anti-TNF. Como os pacientes em uso dessa terapia apresentam risco de infecção pneumocócica letal e não apresentam resposta máxima à vacinação quando em uso deste imunobiológico, sugere-se a vacinação antes de se iniciar a terapia anti-TNF ${ }^{(22,23)}$.

A vacina pneumocócica é, portanto, segura e recomendada em pacientes com doenças reumatológicas, apesar da resposta diminuída de anticorpos em determinados grupos. Devido à possibilidade de redução nos títulos de Ac com o tempo, sugere-se repetir a dose da vacina a cada 2 a 3 anos em pacientes imunodeprimidos ${ }^{(22)}$.

Vacina Haemophilus influenza e tipo B (Hib) - As doenças invasivas causadas pelo $H$. influenza e tipo B em crianças, como meningite, septicemia, epiglotite, tiveram sua incidência reduzida com a introdução da vacinação na infância. A imunidade contra Hib é mediada por anticorpos séricos contra o polissacarídeo capsular da bactéria. A vacina é administrada por via intramuscular (IM), sendo necessárias duas doses com dois meses de intervalo para pacientes imunossuprimidos. Os efeitos adversos são leves: eritema local e reação febril leve ${ }^{(3)}$.

Adultos saudáveis não têm risco de desenvolver infecções por $H$. influenza e, no entanto, pacientes imunocomprometidos podem apresentar septicemia ou infecção localizada.

Existem poucas informações a respeito da imunogenicidade dessa vacina e do risco de exacerbação dos sintomas em pacientes com doenças reumatológicas. Há evidência de que a grande maioria dos pacientes com LES desenvolve níveis de Ac protetores contra $\mathrm{Hib}^{(7)}$.

Vacina meningocócica conjugada tipo $\mathbf{C}-\mathrm{A}$ meningite meningocócica e a septicemia são afecções que podem ocorrer esporadicamente ou em surtos. 
A vacina meningocócica está indicada a partir dos 2 meses de idade, nos portadores de asplenia congênita ou adquirida, deficiências dos componentes finais do complemento, anemia falciforme e talassemia, esplenectomizados.

A vacina contra o meningococo deve ser aplicada IM em dose única. As reações adversas ocorrem no local da aplicação e são eritema, edema e dor, podendo ocorrer febre nos primeiros três dias.

\section{VACINAS CONTRA DOENÇAS VIRAIS}

Vacina contra varicela - A varicela é uma doença predominantemente das crianças, porém $10 \%$ dos adultos são suscetíveis à infecção e, quando desenvolvem a doença, apresentam um risco aumentado para as complicações pulmonares e do sistema nervoso central. Pacientes imunocomprometidos que adquirem a doença primária apresentam um risco de doença grave e morte. Imunização em contactantes domiciliares soronegativos para a infecção e a profilaxia pós-exposição do paciente com imunoglobulina são medidas importantes para pacientes imunocomprometidos ${ }^{(22)}$.

A vacina contra a varicela consiste do vírus varicela zoster vivo atenuado. É segura e eficaz em adultos saudáveis, no entanto não é recomendada em imunocomprometidos, incluindo pacientes com doenças reumatológicas.

Vacina oral (VOP) e inativada (VIP) da poliomielite A poliomielite (pólio) permanece como causa significante de doença e incapacidade em certas regiões do mundo.

A vacina oral contra a pólio (VOP) é composta por vírus vivo, sendo contra-indicada em pacientes imunocomprometidos e contactantes domiciliares. Quando houver indicação de vacinação contra pólio em pacientes imunocomprometidos, seus familiares ou pessoas de contato próximo, estes devem receber a vacina pólio inativada (VIP). Quando do uso inadvertido da vacina oral nos contactantes, recomenda-se evitar o contato com o paciente por aproximadamente um mês. Assim pacientes em uso de drogas anti-TNF devem evitar o contato com crianças ou adultos que receberam a vacina viva durante quatro semanas.

Vacina SCR (sarampo/caxumba/rubéola) - O sarampo e a rubéola ainda ocorrem em áreas endêmicas no mundo e em populações subimunizadas em países desenvolvidos. O sarampo pode causar doença grave em pacientes imunocomprometidos.

A vacina SCR é constituída de vírus vivo, sendo contra-indicada na maioria das pessoas imunocomprometidas, exceto em determinados pacientes infectados pelo HIV.
Além do mais, o Center for Disease Control and Prevention (CDC) admite a administração dessa vacina a indivíduos em uso de corticosteróide a curto prazo, doses baixas ou moderadas de corticosteróide ou com tratamento a longo prazo em dias alternados com preparações de curta ação ou mantidas em doses fisiológicas ${ }^{(23)}$.

Pacientes imunocomprometidos, soronegativos para a infecção, expostos ao sarampo devem receber gamaglobulina IM, administrada até seis dias após a exposição, profilaticamente. A vacina SCR não é transmitida por contactantes, portanto os familiares de pacientes imunocomprometidos devem recebê-la.

A vacina da rubéola é causa bem estabelecida de artrite associada à vacina, especialmente em mulheres adultas. Ocorre uma sinovite transitória nas mãos e nos punhos, que ocasionalmente pode se tornar uma artrite prolongada, porém o estudo de Ray et al. ${ }^{(24)}$ não evidenciou um aumento do risco para artropatia crônica.

Vacina contra influenza - $\mathrm{O}$ vírus da influenza pode causar doença grave ou morte em pacientes imunocomprometidos, quer por conseqüência direta da infecção pelo influenza ou por infecção bacteriana secundária.

A vacina é inativa e consiste em dois tipos, A e B. Está indicada, entre outras situações, pelo Comitê Técnico Assessor de Imunizações do Ministério da Saúde, para pacientes imunocomprometidos e seus familiares contactantes.

A possibilidade de que a vacina influenza possa deflagrar ou exacerbar uma artrite inflamatória é controversa. Os componentes virais incluídos nas vacinas variam ano após ano, com evidências de que algumas vacinas para influenza são associadas com complicações auto-imunes.

O estudo de Brow \& Bertouch $^{(8)}$ evidenciou a presença de LES, polimialgia reumática e artrite reumatóide após a vacinação contra o influenza. A paciente que desenvolveu LES apresentou os primeiros sintomas da doença duas semanas após a vacinação. Quatro meses após a vacinação, apresentou anticorpo antinuclear (ANA) de $1 / 320$ com padrão homogêneo e Ac anti-DNA de dupla hélice (dsDNA) no título de 87\% (normal = 0\%-60\%) e preenchia quatro dos 11 critérios da ARA para o diagnóstico de LES. Seis semanas após o diagnóstico, os sinais e sintomas de LES diminuíram e o ANA era de $1 / 80$ e o Ac anti-dsDNA de 4 UI (normal = 0-7). Nove meses mais tarde, a paciente permanecia assintomática. $\mathrm{O}$ esposo dessa paciente, o qual foi vacinado no mesmo dia, desenvolveu um quadro clínico compatível com polimialgia reumática, três semanas após a vacinação. Quatro meses após a vacinação, apresentava velocidade de hemossedimentação (VHS) de $45 \mathrm{~mm} / \mathrm{h}$, 
sendo feito diagnóstico de polimialgia reumática e tratado com $15 \mathrm{mg}$ de prednisolona, respondendo rapidamente com alívio dos sintomas. O terceiro caso é de uma mulher de 44 anos de idade que apresentou, três dias após a vacinação, um quadro influenza-like e dor, rigidez matinal e edema de mãos, punhos, ombros, tornozelos e joelho esquerdo. Seis meses após a vacinação, apresentava uma sinovite simétrica de pequenas e grandes articulações com velocidade de hemossedimentação (VHS) de $55 \mathrm{~mm} / \mathrm{h} \mathrm{e}$ fator reumatóide num título de 512. Foi feito diagnóstico de artrite reumatóide, preenchendo cinco dos sete critérios da ARA. Paciente entrou em remissão com uso da D-penicilamina. No estudo da Clínica $\mathrm{Mayo}^{(25)}$, porém, não se observou aumento da prevalência de artrite reumatóide (AR) após a vacinação.

Presença de vasculite sistêmica, envolvendo pele, pulmão e rins, vasculite reumatóide, trombocitopenia, uveíte e pericardite, artrite reativa, poliartropatia, miosite orbitária, esclerite posterior e casos ocasionais de falsos testes sorológicos para HIV, HTLV-1 e hepatite C foram relatados após a aplicação da vacina influenza ${ }^{(26-29)}$.

Chalmers et al.$^{(30)}$ compararam a resposta à vacinação de 126 pacientes com AR com a de controles saudáveis. Observaram uma resposta adequada à vacinação, sem evidência de aumento das reações adversas. $\mathrm{O}$ uso da vacina contra influenza nos pacientes com AR permanece controverso, embora evidências sugiram que a incidência de flares possa não ser significante.

Hess et al. (31) $^{(3)}$ obervaram que a vacina contra influenza não causou piora da atividade em pacientes com LES leve; além de que os títulos de anticorpos foram similares entre os pacientes e controles.

Abu-Shakra et al..$^{(32)}$, em um estudo randomizado com 48 pacientes com LES, em que 24 pacientes receberam a vacina e 24 não foram imunizados, verificaram que o SLEDAI não foi significativamente diferente entre os grupos, não ocorrendo atividade da doença entre os pacientes que receberam a vacina. A vacina mostrou ser, portanto, segura. Em um estudo posterior ${ }^{(33)}$, o mesmo autor acompanhou 24 pacientes com LES avaliados 12 dias após a vacinação influenza. Desses pacientes, 17 faziam uso de CE (dose média de $12 \mathrm{mg} /$ dia), nove estavam em uso de hidroxicloroquina e três pacientes, em uso de azatioprina. Houve uma resposta com produção de Ac contra influenza em $75 \%$ dos pacientes, os $25 \%$ restantes que não responderam apresentavam idade média de 50 anos, e todos estavam recebendo CE na dose média de $15 \mathrm{mg} / \mathrm{d}$ e dois pacientes faziam uso de azatioprina. $\mathrm{O}$ número de pacientes que responderam à vacina foi menor do que o esperado entre a população geral.

Segundo Abu-Shakra et al..$^{(32-33)}$, apesar de a resposta imune ser deficiente em alguns grupos, os pacientes com LES devem receber a vacina pelo fato de esta ser segura, bem tolerada e protetora. Embora não haja evidência de que a vacina contra influenza não seja segura em pacientes com LES, não há estudos que avaliem pacientes com doses repetidas da vacina (anuais) ${ }^{(34)}$.

Em pacientes com artrite reumatóide, mesmo em uso de altas doses de imunossupressores, a vacina é segura, porém nesses pacientes a resposta imunogênica em relação aos que estão em uso de baixa dose de imunossupressores é menor ${ }^{(35)}$.

Vacina contra febre amarela - É composta por vírus vivo atenuado.

Recomenda-se o uso da vacinação entre viajantes ou pessoas que moram em áreas endêmicas. Não se deve administrar a vacina em pacientes imunocomprometidos, pois apresentam um risco elevado de encefalite.

Vacina contra hepatite $\mathbf{A}-\mathrm{A}$ infecção pelo vírus da hepatite A é prevalente em alguns países. Viajantes para áreas endêmicas ou pacientes com risco para a infecção têm indicação de receberem a vacina. Está indicada, também, para pessoas com hepatopatias crônicas suscetíveis à hepatite A.

A vacina é imunogênica na população saudável, porém parece ser menos imunogênica em pacientes imunocomprometidos. Na pós-infecção, pode ser feita a profilaxia com imunoglobulina.

Pouca ou nenhuma informação existe em relação à exacerbação de doença reumatológica.

Vacina contra hepatite B - O vírus da hepatite B é causa significante de doença crônica. Pacientes imunocomprometidos têm risco elevado de desenvolver um estado crônico após a infecção aguda pelo vírus da hepatite $\mathrm{B}$. É recomendável o uso da vacina em grupos de risco.

A vacina da hepatite $B$ é considerada segura e eficaz em indivíduos saudáveis, mas pode ter uma resposta diminuída em pacientes transplantados, alcoólatras, diabéticos e outros grupos.

Vários eventos adversos são relatados com o uso dessa vacina, como oclusão da veia central da retina, uveíte, síndrome nefrótica, desmielinização do sistema nervoso central e ou$\operatorname{tros}^{(36)}$. Várias manifestações reumatológicas têm sido relatadas após vacinação contra hepatite $B$, incluindo artrite reumatóide, 
exacerbação de LES não diagnosticado previamente, artrite pós-vacinal, poliartralgia, mialgia e vasculite ${ }^{(12)}$.

$A$ presença de $A R$ após a vacinação contra hepatite $B$ tem sido descrita em vários estudos, sugerindo uma relação causal entre estas. Um desses estudos ${ }^{(13)}$ descreveu 11 casos de AR após a vacinação de rotina; todos os pacientes eram saudáveis antes da vacinação e, após esta, apresentaram os critérios do America College of Rheumatology (ACR) para AR. Esses pacientes foram seguidos durante dois anos. Nove dos 11 pacientes apresentavam HLA classe II - shared epitope, mostrando que fatores genéticos podem representar um risco para artrite após a vacinação contra a hepatite B.

Desenvolvimento de síndrome de Sjögren foi demonstrado um mês após a primeira dose da vacina da hepatite B em uma paciente, a qual apresentou poliartralgias, sinais objetivos de olho seco, evidência histopatológica de sialadenite e presença de Ac anti-Ro, satisfazendo os critérios para síndrome de Sjögren. Entretanto, permanece incerto se a vacina da hepatite $B$ apresenta um efeito direto nos achados clínicos e laboratoriais da síndrome de Sjögren; contudo, a história prévia de vacinação contra hepatite $\mathrm{B}$ deve ser questionada em pacientes com sintomas clínicos recentes de síndrome de Sjögren ${ }^{(37)}$.

No estudo realizado por Elkayam et al. ${ }^{(38)}, 44$ pacientes com AR foram randomizados, 22 pacientes receberam três doses da vacina contra hepatite $\mathrm{B}$ e 22 não receberam a vacina; nenhum paciente apresentou efeito adverso à vacina nem piora significativa nas medidas laboratoriais e clínicas. A resposta humoral nos pacientes foi reduzida, sendo que apenas $68 \%$ destes responderam à vacinação após seis meses, com níveis de Ac >10 UI/l, comparado com a resposta humoral esperada superior a $85 \% \mathrm{em}$ adultos jovens. Essa resposta humoral reduzida foi associada com idade avançada e com dor intensa durante o dia, não sendo associada ao uso de prednisona, MTX, azatioprina, hidroxicloroquina, ouro intramuscular, antiinflamatório não-hormonal, nem ao número de juntas inflamadas ou dolorosas, VHS, nem PCR (proteína C reativa). Esse estudo sugere que a imunização deve ser preferencialmente administrada quando a atividade da doença é baixa, pois a atividade da doença avaliada pela presença de dor intensa durante o dia correlacionou-se com a não resposta à imunização. A vacinação contra hepatite $B$, nessa coorte, foi segura e imunogênica para a maioria dos pacientes, embora um subgrupo tenha permanecido exposto à infecção pelo vírus da hepatite $\mathrm{B}$ a despeito da imunização.

A vacina contra a hepatite $B$ mostrou ser segura e eficaz em muitos pacientes com doenças reumáticas, devendo ser administrada em três doses, sendo o intervalo entre a primeira e segunda dose de um mês e a terceira dose deve ser administrada seis meses após a primeira. Nos pacientes com LES, entretanto, a segurança da vacinação contra hepatite $B$ não está determinada. A British Society of Rheumatology recomenda que pacientes com LES e risco de exposição à hepatite $B$ devam receber a vacina.

\section{CONCLUSÃO}

Infecção é freqüente entre pacientes com doenças reumatológicas auto-imunes e/ou terapia imunossupressora, sendo uma das principais causas de morte nesses pacientes. A realização da imunização passiva é, pois, uma estratégia importante e segura para evitar as infecções e, portanto, diminuir a letalidade.

Embora vários trabalhos mostrem que uma parte dos pacientes com doenças reumatológicas e/ou em uso de terapia imunossupressora não apresenta resposta de Ac satisfatória à vacinação, devido não só à própria doença, mas também pela terapia imunossupressora, a eficácia da vacinação, comprovada pelo título de Ac na maioria dos pacientes, justifica a imunização.

A exacerbação das doenças quando submetidos à vacinação ocorre em uma porcentagem muito pequena de pacientes, o que nos encoraja à imunoprevenção, especialmente das doenças preveníveis por vacinas compostas por bactérias e vírus mortos, as quais são muito seguras, mesmo na vigência do uso de corticoterapia em dose alta ou terapias imunossupressoras. Algumas vacinas, especialmente contra hepatite B e SCR, podem deflagrar doença auto-imune.

Vacinas com bactérias ou vírus vivos atenuados são, em geral, contra-indicadas em pacientes imunossuprimidos.

\section{AGRADECIMENTO}

À enfermeira Neusa Perrela, pela revisão dos textos referentes a composição, doses, intervalos e vias de administração das vacinas.

Declaramos a inexistência de conflitos de interesse. 


\section{REFERÊNCIAS}

1. Lockshin MD: Vaccinations and Rheumatic Disease, 2001. Disponível em: <www.rheumatology.hss.edu>.

2. Robin KA: Vaccination of Imunossupressed Adult Patient with Rheumatologic disease. Rheum Dis Clin North Am 25: 567-83, 1999.

3. Ioannou Y, Sultan S, Isenberg DA: Causes of death in SLE: analysis of 266 patients under long term review. Ann Rheum Dis (EULAR Abstract 665 ), 1999.

4. Portaria $\mathrm{GM} \mathrm{n}{ }^{\circ} 597$, artigo $2^{\circ}$, de 8 de abril de 2004 .

5. Jenner E: Department of Health, Welsh Office, Scottish Office Department of Health, DHSS (Northern Ireland): Immunization against infectious disease. Bicentenary ed. London: HMSO, 1996.

6. Tishler M, Shoenfeld Y: Vaccination may be associated with autoimmune diseases. Isr Med Assoc J 6(7): 430-2, 2004.

7. Battafarano DF, Battafarano NJ, Larsen L, et al: Antigen specific antibody responses in lupus patients following immunization. Arthritis Rheum 41: 1828-34, 1998.

8. Brow MA, Bertouch JV: Rheumatic complications of influenza vaccination. Aust N Z Med 24: 572-3, 1994.

9. Schattner A, Ben-Chetrit E, Schmilovitz H: Poliovaccines and the course of systemic lupus erythematosus - a retrospective study of 73 patients. Vaccine 10: 98-100, 1996.

10. Older SA, Battafarano DF, Enzenauer RJ, et al: Can immunization precipate connective tissue disease? Report of five cases of systemic lupus erythematosus and review of the literature. Semin Arthritis Rheum 29: 131-9, 1999.

11. Aron-Maor A, Shoenfeld Y: Vaccination and systemic lupus erythematosus; the bidirectional dilemmas. Lupus 10: 237-40, 2001.

12. Maillefert JF, Sibilia J, Toussirot E, et al: Rheumatic disorders developed after hepatitis B vaccination. Rheumatology 38: 978 83, 1999.

13. Pope JE, Stevens A, Howson W, Bell DA: The development of rheumatoid arthritis after recombinant hepatitis $\mathrm{B}$ vaccination. J Rheumatol 25: 1687-93, 1998.

14. American College Physicians Task Force on Adult Immunization and Infectious Diseases Society of America: Guide for Adult Immunization. Philadelphia. American College of Physicians, 1984.

15. Symmons DPM, Chakravarty K: Can immunization trigger rheumatoid arthritis? Ann Rheum Dis 52: 843-4, 1993.

16. Harrison BJ, Thomson W, Pepper L, et al: Patients who develop inflamatory polyarthritis (IP) after immunization are clinically indistinguishable from other patients with IP. Br J Rheumatol 36: 366-9, 1997.

17. Prevention of Pneumococcal Disease. Recomendations of the Advisory Comitte on Immunization Practice (ACIP) MMWR Morb Mortal Wkly Rhep 46(RR-8): 1-24, 1997.

18. Elkayam O, Paran D, Caspi D, et al: Immunogenicity and safety of pneumococcal vaccination in patients with rheumatoid arthritis or systemic lupus erythematosus. Clin Infect Dis 34: 147-53, 2002.

19. Tarjan P, Sipka S, Marodi L, et al: No short-term immunological effects of Pneumococcus vaccination in patients with systemic lupus erythematosus. Scand J Rheumatol 21: 211-5, 2002.

20. O'Dell J, Gilg J, Palmer W, et al: Pneumococcal vaccination: decresead antibody response in the rheumatoid arthritis patients on methotrexate. Arthritis Rheum 35(Suppl 9): S197, 1992.
21. Elkayam O, Caspi D, Reitblatt T, Charboneau D, Rubins JB: The effect of Tumor Necrosis Factor Blockade on the response to pneumococcal vaccination in patients with rheumatoid arthritis and ankylosing spondylitis. Semin Arthritis Rheum 33: 283-8, 2004.

22. Center for Disease Control and Prevention: Prevention of Varicella - recomendation of the Advisory Comitee on Immunization Practice (ACIP). MMWR Morb Mortal Wkly Rep, 45(RR-11): 1-36, 1996.

23. Center for Disease Control and Prevention: Update: Vaccine side effects, adverse reactions, contraindications, and precautions - recomendation of the Advisory Comitee on Immunization Practice (ACIP). MMWR Morb Mortal Wkly Rep, 45(RR-12): 1-35, 1996.

24. Ray P, Black S, Shinefield H, et al: Risk of chronic arthropathy among women after rubeolla vaccination. JAMA 278: 551-6, 1997.

25. Kurland LT, Molgaard CA, Kurland EM, Erdtmann FJ, Stebbing GE: Lack of association of swine flu vaccine and rheumatoid arthritis. Mayo Clin Proc 59: 816-21, 1984.

26. Mackenzie WR, Davis JP, Peterson DE, et al: Multiple falsepositive serologic tests for HIV, HTLV-1, and hepatitis C following influenza vaccination. JAMA 268: 1015-7, 1992.

27. Thurairajan G, Hope-Ross MW, Situnayake RD, et al: Polyarthropaty, orbital myositis, and posterior scleritis. An usual adverse reaction to influenza vaccine. Br J Rheumatol 36: 120-3, 1997.

28. Mader R, Narendran A, Lewtas J, et al: Systemic vasculitis following influenza vaccination - report of 3 cases and literature review. J Rheumatol 20: 1429-1, 1993.

29. Karan PI, Limaye V, Hill, et al: Rheumatoid Vasculitis following influenza vaccination. Rheumatol 42(7): 907-9, 2003.

30. Chalmers A, Scheifele D, Patterson C, et al: Immunization of patients with rheumatoid arthritis against influenza: A study of vaccine safety and immunogenicity. J Rheumatol 21: 1203-6, 1994.

31. Hess EV, Hahn B: Influenza immunization in lupus erythematosus: safe, effective? (editorial). Ann Intern Med 88: 833-4, 1978.

32. Abu-Shakra M, Zalmanson S, Neumann L, Flusser D, Sukenik S, Buskila D: Influenza virus vaccination of patients with systemic lupus erythematosus: effects on disease activity. J Rheumatol 27: 1681-5, 2000.

33. Abu-Shakra M, Press J, Varsano N, et al: Specific Antibody Response After Influenza Immunization in SLE. J Rheumatol 29: 2555-7, 2002.

34. Ioannou $Y$, Isenberg DA: Immunization of patients with sistemic lupus erytematosus: the current state of play. Lupus 8: 497-501, 1999.

35. Bridges MJ, Coady D, Kelly CA, Hamilton J, Heycock C: Factors influencing uptake of influenza vaccination in patients with rheumatoid arthritis. Ann Rheum Dis 62(7): 685, 2003.

36. Anonymous. Update: vaccine side effects, adverse reactions, contraindications and precautions. Recomendations of the advisory committee on immunization practices (ACIP). MMWR Morb Mortal Wkly Rep 45: 1-35, 1996.

37. Toussirot E, Lohse A, Wendling D, Mougin C: Sjogren's syndrome occurring after hepatitis B vaccination. Arthritis Rheumatol 43: 2139-40, 2000.

38. Elkayam O, Yaron M, Caspi D: Safety and efficacy of vaccination against hepatitis $\mathrm{B}$ in patients with rheumatoid arthritis. Ann Rheum Dis 61: 623-5, 2002. 